\title{
Structure of the Tripartite Multidrug Efflux Pump AcrAB-TolC Suggests an Alternative Assembly Mode
}

\author{
Jin-Sik Kim ${ }^{2,6}$, Hyeongseop Jeong ${ }^{3,6}$, Saemee Song ${ }^{1,4}$, Hye-Yeon Kim ${ }^{5}$, Kangseok Lee ${ }^{4}$, Jaekyung Hyun ${ }^{3, *}$, \\ and $\mathrm{Nam}-\mathrm{Chul} \mathrm{Ha}{ }^{1, *}$
}

\begin{abstract}
Escherichia coli AcrAB-TolC is a multidrug efflux pump that expels a wide range of toxic substrates. The dynamic nature of the binding or low affinity between the components has impeded elucidation of how the three components assemble in the functional state. Here, we created fusion proteins composed of AcrB, a transmembrane linker, and two copies of AcrA. The fusion protein exhibited acridine pumping activity, suggesting that the protein reflects the functional structure in vivo. To discern the assembling mode with ToIC, the AcrBA fusion protein was incubated with TolC or a chimeric protein containing the TolC aperture tip region. Three-dimensional structures of the complex proteins were determined through transmission electron microscopy. The overall structure exemplifies the adaptor bridging model, wherein the funnel-like AcrA hexamer forms an intermeshing cogwheel interaction with the $\alpha$-barrel tip region of TolC, and a direct interaction between AcrB and TolC is not allowed. These observations provide a structural blueprint for understanding multidrug resistance in pathogenic Gram-negative bacteria.
\end{abstract}

\section{INTRODUCTION}

Multidrug resistance in pathogenic bacteria is one of the greatest threats to human health (Nikaido and Zgurskaya, 1999).

\footnotetext{
${ }^{1}$ Department of Food and Animal Biotechnology, Department of Agricultural Biotechnology, Center for Food and Bioconvergence, Research Institute for Agricultural and Life Sciences, Seoul National University, Seoul 151-742, Korea, ${ }^{2}$ Department of Manufacturing Pharmacy, Pusan National University, Busan 609-735, Korea, ${ }^{3}$ Division of Electron Microscopic Research, Korea Basic Science Institute, Dajeon 169-148, Korea, ${ }^{4}$ Department of Life Science, Chung-Ang University, Seoul 156-756, Korea, ${ }^{5}$ Division of Magnetic Resonance, Korea Basic Science Institute, Chungbuk $363-883$, Korea, ${ }^{6}$ These authors contributed equally to this work.

*Correspondence: hanc210@snu.ac.kr (NCH); hjk002@kbsi.re.kr (JH)
}

Received 17 October, 2014; revised 17 November, 2014; accepted 18 November, 2014; published online 15 January, 2015

Keywords: complex structure, electron microscopy, membrane protein, multidrug efflux pump
AcrAB-TolC is the maior multidruq efflux pump in Escherichia coli, and its overexpression is common in multidrug-resistant clinical isolates (Morshed et al., 1995). This drug efflux pump is composed of three essential components: AcrA, AcrB, and TolC (Lewis, 2000). The homotrimeric AcrB belongs to the resistance-nodulation-division (RND) family of proteins, is embedded in the inner membrane, and pumps out diverse substances using a proton gradient as an energy source (Lewis, 2000; Murakami et al., 2002). Crystal structures of AcrB show the molecular mechanisms underlying substrate transport through proton-motif energy (Murakami et al., 2002; Seeger et al., 2006). TolC is also homotrimeric membrane protein that displays a long cylinder-like structure, which provides a conduit across the outer membrane (Koronakis et al., 2000). AcrA is an adaptor protein that connects $\mathrm{AcrB}$ and TolC in the periplasm (Fralick, 1996; Ma et al., 1993). The TolC channel remains closed without the other components, and channel opening is initiated by binding to AcrA and/or AcrB (Koronakis et al., 2000). Several structures have been determined for AcrA and its homologues MexA in the Pseudomonas aeruginosa MexABOprM pump as well as MacA in the macrolide-specific drug efflux pump MacAB-TolC pump, which show that AcrA consists of an $\alpha$-helical domain, lipoyl domain, $\beta$-barrel domain, and membrane-proximal domain (Mikolosko et al., 2006; Symmons et al., 2009). However, the oligomeric state of AcrA in the functional complex is controversial.

Binding between AcrA and TolC as well as between AcrA and AcrB is likely dynamic. The binary complexes AcrA - TolC and AcrB - AcrA were observable albeit with low affinity. However, the direct interaction between AcrB and TolC is elusive (Touze et al., 2004). The interaction between AcrB and TolC was detected when binding was fixed through cross-linking (Tamura et al., 2005). Based on this direct interaction between AcrB and TolC, an 'adapter wrapping model' was proposed for AcrAB-TolC pump assembly and supported by extensive binding mapping using Cys-mediated cross-linking experiments (Lobedanz et al., 2007; Symmons et al., 2009; Tamura et al., 2005). The key feature of the adapter wrapping model is that a tip-to-tip interaction between AcrB and TolC and three AcrA protomers wraps the outer portion of the AcrB and TolC binary complex with a stoichiometry of 3:3:3 (Hinchliffe et al., 2013).

The stoichiometry and binding assembly of the AcrAB-TolC pump has been under debate. Funnel-shaped hexmeric assembly of the adaptor protein was observed in the MacA crystal 
structure (Rouquette-Loughlin et al., 2005; Yum et al., 2009). Based on this structure, we proposed an 'adaptor bridging model', where the apical tip of the $\alpha$-barrel consisting of six $\alpha$ hairpins from the AcrA hexamer forms an intermeshing cogwheel interaction with the analogous six-bladed cogwheel of an $\alpha$-barrel in the TolC trimer (Xu et al., 2011a; Yum et al., 2009). This model was further supported by extensive structural, biochemical, and genetic studies (Janganan et al., 2011; Kim et al., 2010; Xu et al., 2010; 2011a; 2011b; 2012; Yum et al., 2009). Structures of a chimeric protein containing the $\alpha$-hairpin domain of MexA show a funnel-like hexameric arrangement (Xu et al., 2011a). In an electron microscopy study on a complex protein containing the adaptor protein and TolC, an intermeshing cogwheel interaction between the $\alpha$-barrel tip regions of the adaptor protein and TolC was exhibited (Xu et al., 2010; 2011a; 2012). Independent research groups presented evidence for the pump assembly with stoichiometry among the components (Janganan et al., 2011; Mima et al., 2007; Narita et al., 2003; Su et al., 2011).

In this study, we provide structures of the AcrAB-TolC pump using the AcrBA fusion protein. Our structure exemplifies the adaptor bridging model based on an intermeshing cogwheel interaction between the AcrA hexamer and TolC. Very recently, a complex structure of the AcrAB-TolC pump was reported, which essentially describes the same density envelop for the complex but exhibits a different binding interface between AcrA and TolC in their docked model (Du et al., 2014). Here, we compare the two models and discuss the confidence of the previous results compared with the adaptor-bridging model.

\section{MATERIALS AND METHODS}

\section{Constructing the fusion proteins}

To construct plasmids for the AcrBA fusion protein (AcrB-TM\#AcrA-AcrA) and measure the activity, three DNA fragments were sequentially inserted into the plasmid. First, DNA fragments encoding full-length $E$. coli AcrB were inserted into the pET22b vector (Novagen) Ndel and Hindlll sites, which produced the vector $\mathrm{pET} 22 \mathrm{~b}-\mathrm{AcrB}$. DNA fragments encoding AcrA (residues 26-397), the Ser3 linker, and AcrA (residues 26-397 without the stop codon) were generated using the overlapping PCR technique. The DNA fragments were inserted into the pET22b-AcrB vector Hindlll and Xhol sites, which produced the vector pET22b-AcrB-AcrA-AcrA. Lastly, each TM region (Supplementary Table S1) was inserted between the AcrB and AcrA dimer using the vector pET22b-AcrB-AcrA-AcrA Hindlll site through the Cold Fusion ligation method in accordance with the manufacturer's instruction, which yielded the vectors $p E T 22 b$ AcrB-TM\#(1-6)-AcrA-AcrA. We added the Precision protease recognition sequence (LEVLFQGP) and Streptococcus dysgalactiae Protein G (residues 302-427) to the AcrBA fusion protein $\mathrm{C}$-terminus to facilitate purification through affinity chromatography. To add this sequence, a DNA fragment encoding the PreScission protease cleavage site as well as Protein $\mathrm{G}$ was synthesized (Bioneer, Korea) and inserted into the pET22bAcrB-TM\#(1-6)-AcrA-AcrA Xhol site using the Cold Fusion ligation method, which generated $p E T 22 b-A c r B-T M \# 5-A c r A-$ AcrA-ProG. To produce MacA-TolC $\alpha$-hybrid-dimer, the previously described plasmid pPRO-MacA-TolC $\alpha$-hybrid-dimer (Xu et al., 2011b) was used. To produce the E. coli TolC protein, DNA fragments encoding E. coli TolC 1-452 (Koronakis et al., 2000) were inserted into the pET28a vector Ncol and Xhol sites, which produced the vector $\mathrm{pET} 28 \mathrm{a}-\mathrm{TolC}$. Sequences of the primers used in this study are listed in Supplementary Table S2.
Acridine pumping assay

The pET22b-AcrB-TM\#(1-6)-AcrA-AcrA plasmids were transformed into the $E$. coli strain BW25113 (DE3) $\triangle a c r A B$, which was generated from the BW25113 $\triangle a c r A B$ strain (Yum et al., 2009) using the $\lambda$ DE3 Ivsogenization kit (Merk, Germanv). The acridine pumping assay was performed using the $E$. coli strains and based on the procedure previously described (Martins and Amaral, 2012). For the positive control, the $a c r A B$ gene cluster was inserted into $\mathrm{pET} 22 \mathrm{~b}$.

\section{Minimum inhibitory concentration}

The procedure was previously described (Kim et al., 2009).

Transmission electron microscopy and Image processing Five microliters of the AcrB-TM\#5-AcrA-AcrA or MacA-TolC $\alpha$ hybrid-dimer complex protein at approximately $6 \mu \mathrm{g} / \mathrm{ml}$ was applied to a 300-mesh EM-grid covered with continuous carbon film. After $60 \mathrm{~s}$ of sample adsorption, the grid was washed 5 times using droplets of deionized water followed by negative staining with $5 \mu \mathrm{l}$ of $0.75 \%$ uranyl formate and blotting excess solution with filter paper. The sample was visualized using a Tecnai $\mathrm{G}^{2}$ Spirit TWIN (FEI) transmission electron microscope operated at $120 \mathrm{kV}$. The images were recorded using an Ultrascan4000 charged-coupled device (CCD) camera (Gatan) under low-dose conditions at the nominal magnification $\mathrm{x}$ 52,000 and 0.8-1.0 $\mu \mathrm{m}$ under focus.

\section{Model docking}

The fully open TolC structure was constructed as described previously (Xu et al., 2011b). Briefly, residues 106-187 and 324-404 were moved through riaid bodv movement onto the corresponding region of the $E$. coli MacA structure (PDB code 2FPP; residues 103-177), and the backbone conformations was adjusted to produce an $\alpha$-hairpin structure. To construct the AcrA hexamer model, we first used the partial AcrA hexamer model that was previously reported and contains the $\beta$ barrel domain, lipoyl domain, and $\alpha$-hairpin domain (Xu et al., 2011a). The AcrA membrane proximal domain (residues 26-52 and 298-397) derived from the MexA structure (PDB code 2V4D; residues 13-27 and 264-339) (Symmons et al., 2009) was attached to the partial AcrA hexamer model as a template of the MacA hexamer crystal structure (PDB code 4DKO) (Xu et al., 2012). The MacA-TolC $\alpha$-hybrid dimer was constructed as described previously (Xu et al., 2011b). To dock the structures into the EM reconstructions, the AcrA hexamer and fully open TolC (or the MacA-TolC $\alpha$-hybrid-dimer) structures were regarded as individual rigid bodies. These three rigid bodies were automatically fitted into the EM map through sequential fitting using the program UCSF Chimera (Goddard et al., 2007) and then manually adjusted into the map using the program COOT (Emsley and Cowtan, 2004).

Expression and purification of the efflux pump assembly The construct pET22b-AcrB-TM\#5-AcrA-AcrA-ProG was transformed into $E$. coli strain BL21(DE3) $\triangle a c r A B$. The cells were induced with $0.1 \mathrm{mM}$ ITPG at $18^{\circ} \mathrm{C}$ overnight, harvested and resuspended in lysis buffer 1 (20 mM HEPES $(\mathrm{pH} 7.5), 10 \%$ glycerol, $1 \mathrm{mM}$ PMSF, and $1 \mathrm{mM}$ EDTA). The cells were disrupted using a continuous cell disruptor (Constant Systems, UK), and the cell debris was removed through centrifugation at $10,000 \mathrm{~g}$ for $30 \mathrm{~min}$. The cell lysate was ultracentrifuged at $100,000 \mathrm{~g}$ for $3 \mathrm{~h}$, and the membrane fraction (pellet) was obtained. The membrane fraction was solubilized in $40 \mathrm{ml}$ of lysis buffer 2 [20 mM HEPES (pH 7.5), 10\% glycerol, 1 mM PMSF, 
and $2 \%$ dodecylmaltoside (DDM)] and then dialyzed against $800 \mathrm{ml}$ of a buffer containing $20 \mathrm{mM}$ HEPES (pH 7.5) and 10\% glycerol to reduce the DDM concentration. The AcrBA fusion protein was purified using bovine IgG-coupled resin. The protein sample was incubated with bovine IgG-coupled resin and subsequently washed with washing buffer 1 [20 mM HEPES (pH 7.5), $10 \%$ glycerol, $0.02 \% \mathrm{DDM}$, and $300 \mathrm{mM} \mathrm{NaCl}$ ] and washing buffer 2 [20 mM HEPES (pH 7.5), 10\% glycerol, $0.02 \% \mathrm{DDM}$, and $150 \mathrm{mM} \mathrm{NaCl}$. Next, the purified TolC or MacA-TolC $\alpha$-hybrid-dimer protein was incubated at $30^{\circ} \mathrm{C}$ for 1 $\mathrm{h}$ to allow the proteins to form a complex in the resin. After sequential washing with washing buffer 1 and 2, PreSission protease was added overnight at room temperature in the presence of $2 \mathrm{mM}$ 2-mercaptoethanol. The complex proteins were eluted with washing buffer 2 and then concentrated to $1 \mathrm{mg} / \mathrm{ml}$ using a Centricone concentrator.

\section{Image processing}

Electron micrographs with the pixel size $2.1 \AA$ at specimen scale were used for image processing. Particle images were manually selected and boxed in $256 \times 256$ pixel boxes using the e2boxer.py routine in the EMAN2 software suite (Tang et al., 2007). A total of 2,808 and 1,653 boxed particle images were subsequently processed for class averaging and $3 D$ reconstruction of the AcrB-TM\#5-AcrA-AcrA and MacA-TolC $\alpha$ hybrid-dimer complexes, respectively. Contrast transfer function (CTF) correction of the particle images was performed using e2ctf.py followed by alignment and classification. Next, a reference-free initial 3D model was generated using e2initialmodel.py. The final 3D reconstruction was generated using e2refine_easy.py. Imposing c3 symmetry, the initial model was iteratively refined through projection matching until no further improvement in resolution was observed. A total of 2,591 and 1,563 particle images contributed to the final reconstructions of the AcrB-TM\#5-AcrA-AcrA and MacA-TolC $\alpha-$ hybrid-dimer complexes, respectively. The reconstruction resolution was estimated using Fourier shell correlation 0.5 criteria.

\section{RESULTS}

\section{Construction of the AcrBA fusion proteins}

A major barrier to structural work on the AcrAB-TolC pump was low affinity between the proteins. Moreover, the detergents required to stabilize the membrane proteins may obstruct the protein-protein interactions. To overcome this obstacle, we created fusion proteins that contain AcrA and AcrB. Evidence supporting a 3:6:3 ratio for AcrB:AcrA:TolC has accumulated; however, the functional assembly stoichiometry remains controversial (Janganan et al., 2011; Mima et al., 2007; Narita et al., 2003; Stegmeier et al., 2006; Su et al., 2011; Xu et al., 2009; 2010; 2011a; 2011b; 2012; Yum et al., 2009). As supporting evidence for the AcrA hexamer, an AcrA dimer (two AcrA proteins fused in a single polypeptide chain) exhibited the activity as high as the wild type AcrA (Xu et al., 2011a). To drive the binding and satisfy the stoichiometry between AcrB and AcrA, we designed a long fusion protein, wherein the functional AcrA dimer was fused to the $\mathrm{C}$-terminus of AcrB. Because the AcrB C-terminus is located in the cytoplasmic space (Murakami et al., 2002), and the AcrA is anchored to the perplasmic face of the inner membrane, a transmembrane linker was required between AcrB and the AcrA dimer to bring the AcrB C-terminus to the periplasmic space. Six candidates for the transmembrane linker were selected from transmembrane helices (TM) of other known transmembrane proteins, which yielded the arrange-
A

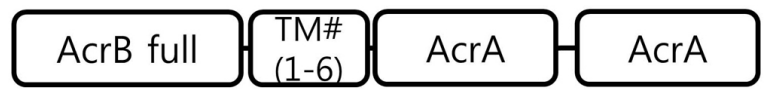

B

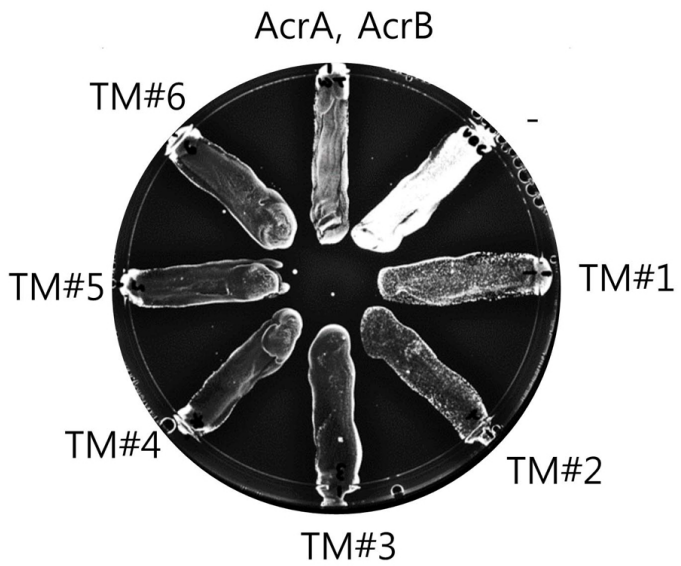

Fig. 1. Construction of the AcrBA fusion proteins and acridine pumping activities. (A) Schematic representations of the construction of the AcrBA fusion protein. (B) Acridine pumping activities of the AcrBA fusion proteins. Each $E$. coli strain BW25113(DE3) $\triangle a c r A B$ transformed with $\mathrm{pET} 22 \mathrm{~b}-\mathrm{AcrB}-\mathrm{TM} \#(1-6)$-AcrA-AcrA was cultured on an acridine-containing LB agar plate, and the pumping activities of the fusion proteins were measured. The $E$. coli strain with the $\mathrm{pET} 22 \mathrm{~b}$ vector containing the wild type AcrA and AcrB genes (AcrA, AcrB) as well as the empty pET22b vector (2) were used as controls.

ment AcrB-TM\#(1-6)-AcrA-AcrA (Fig. 1A and Supplementary Table S1).

To test the activity of these large fusion proteins, we measured the acridine pumping activity using a simple assay on an acridine-containing LB agar plate (Martins and Amaral, 2012) (Fig. 1B). Four proteins displayed strong acridine pumping activity. We used a fusion protein with the TM\#5 sequence and subsequently performed analyses. The minimum inhibitory concentration (MIC) values of the AcrBA fusion protein (herein, AcrB-TM\#5-AcrA-AcrA) were further measured in E. coli toward antibiotics (novobiocin and erythromycin), which showed that the fusion protein had significant activity, albeit lower than the wild type genes (Supplementary Table S3). These results indicate that the forced interaction between AcrB and the AcrA dimer vields a functional assembly and suggests that the interaction recruits TolC in vivo.

Electron microscopy analyses of the AcrBA fusion protein and TolC

To efficiently isolate the fusion protein AcrB-TM\#5-AcrA-AcrA, we fused Protein $\mathrm{G}$ to the $\mathrm{C}$-terminus of the fusion protein with the PreScission protease recognition site. The protein was purified using bovine IgG-coupled resin and incubated on the resin with the independently purified TolC protein. Next, the protein complex was eluted from the resin by cleaving the protease recognition site. The resulting protein complexes were then subjected to negative-staining electron microscopy (EM), which readily identified rocket-like particles (Fig. 2A). Subsequent class averaging enhanced visualization of the particles in 


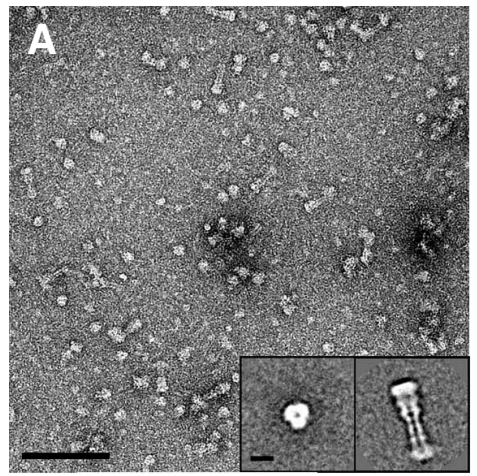

C

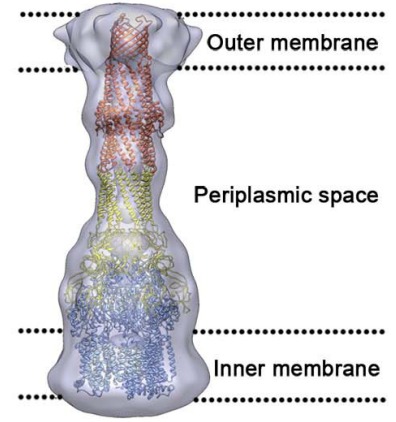

B

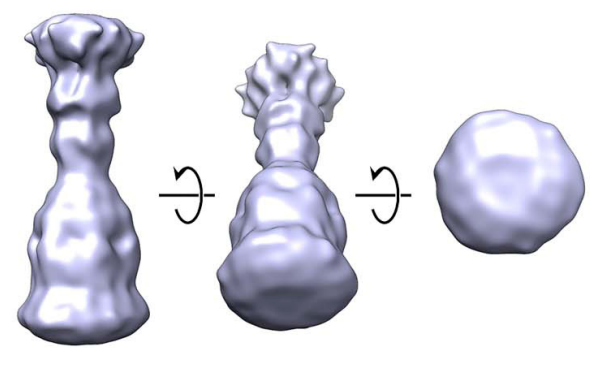

D
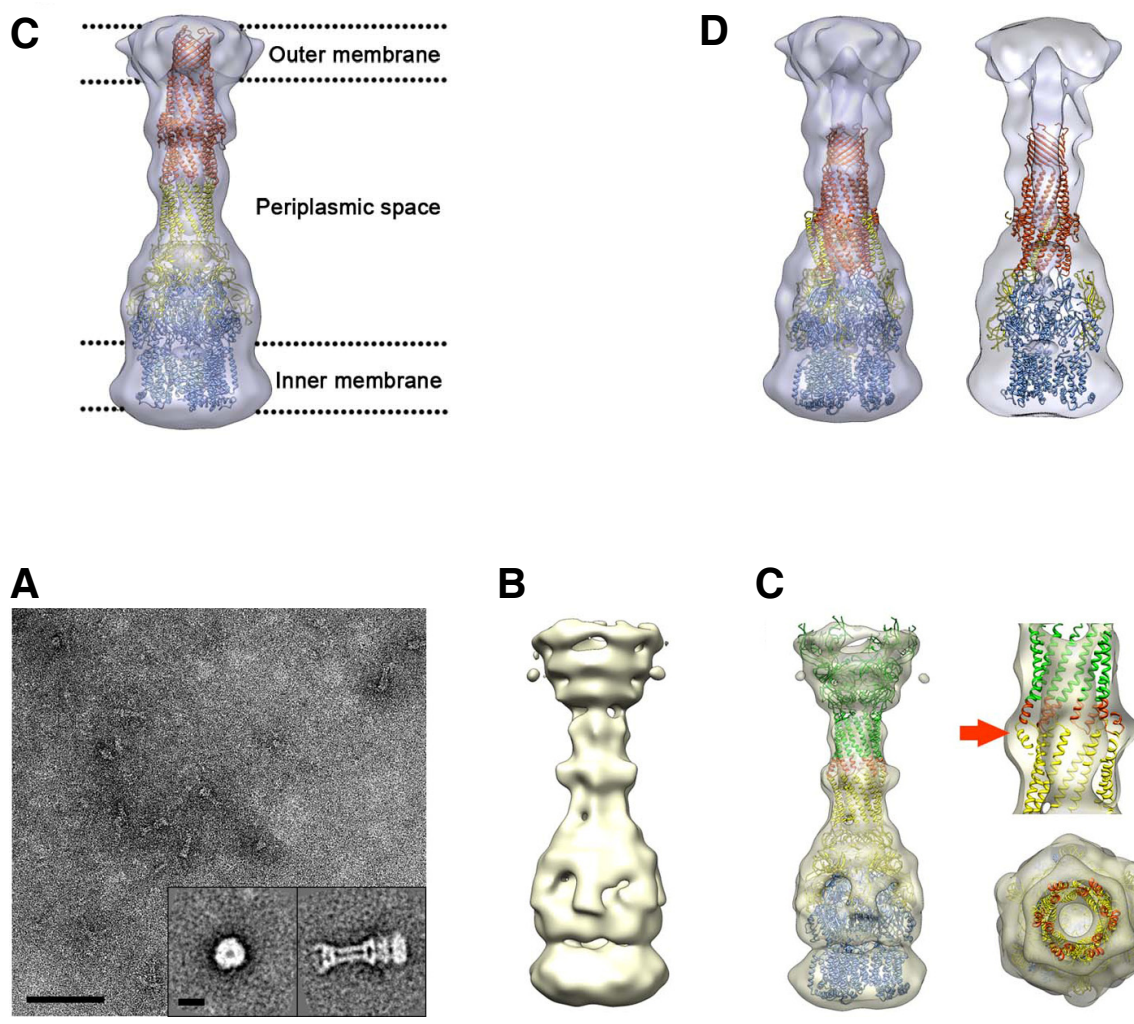

B

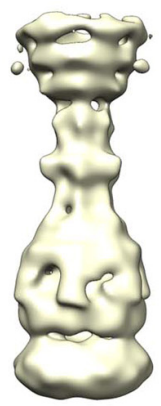

C

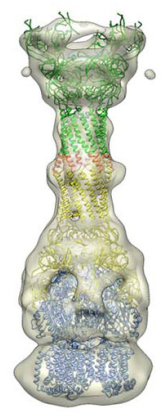

Fig. 2. TEM images and a docked model of the AcrAB-TolC efflux pump. (A) A raw electron micrograph of the purified AcrBA fusion protein and TolC complex as well as representative class averages (inset). (B) 3D reconstruction of the complex. (C) Docked pseudoatomic model of the ternary complex that encompasses the inner membrane (IM), outer membrane (OM), and periplasmic space. (D) Docking the "adaptor wrapping model" into the $3 \mathrm{D}$ reconstruction and the section along the symmetry axis. AcrB is colored blue, AcrA is in yellow, and TolC is in red. The scale bars are $100 \mathrm{~nm}$ and $10 \mathrm{~nm}$ in $(\mathrm{A})$ and the inset, respectively.

and MacA is in green. The dilated binding interface between AcrA and the TolC $\alpha$-hairpin tip is indicated by an arrow. The scale bars are 100 $\mathrm{nm}$ and $10 \mathrm{~nm}$ in (A) and the inset, respectively.

various orientations, from which a three-fold symmetry was apparent along the longest axis. These data are consistent with the notion that AcrB and TolC have a three-fold symmetry, and AcrA has been predicted to have a three- or six-fold symmetry. Accordingly, the 3D density map was produced with a threefold symmetry imposed (Fig. 2B).

At a moderate $26 \AA$, the EM map showed an elongated feature with the AcrB trimer, AcrA hexamer, and TolC trimer (Fig. 2C). To dock the crystal structures of TolC, AcrA, and AcrB, we created a AcrA hexamer model that includes the membrane proximal domain and used the structural model of TolC in the fully-opened conformation (Xu et al., 2011b) as well as the AcrB crystal structure (Murakami et al., 2002). The structures were fitted into the EM map with manual adjustments using rigid body movement; the results were consistent with the den- sity envelop (Fig. 2C). The docked model of the AcrAB-TolC pump has a $770 \mathrm{kDa}$ protein mass and spans the inner membrane, periplasmic space, and outer membrane; it is similar to the modelled structures that exemplify the adaptor bridging model (Xu et al., 2011a; 2011b; 2012; Yum et al., 2009). The cross-section through the 3D-reconstituted density map and docked model exhibit a long channel that runs from the substrate exit region on top of the AcrB to the external medium via the central channels of AcrA and TolC, which is consistent with the homologous MexAB-OprM pump dimensions (Trepout et al., 2010; Xu et al., 2012). However, a docked structure based on the adaptor wrapping model were contradicted to the EM map (Fig. 2D), which demonstrates that the adaptor wrapping model is not consistent with the experimental data for the AcrAB-TolC pump. 

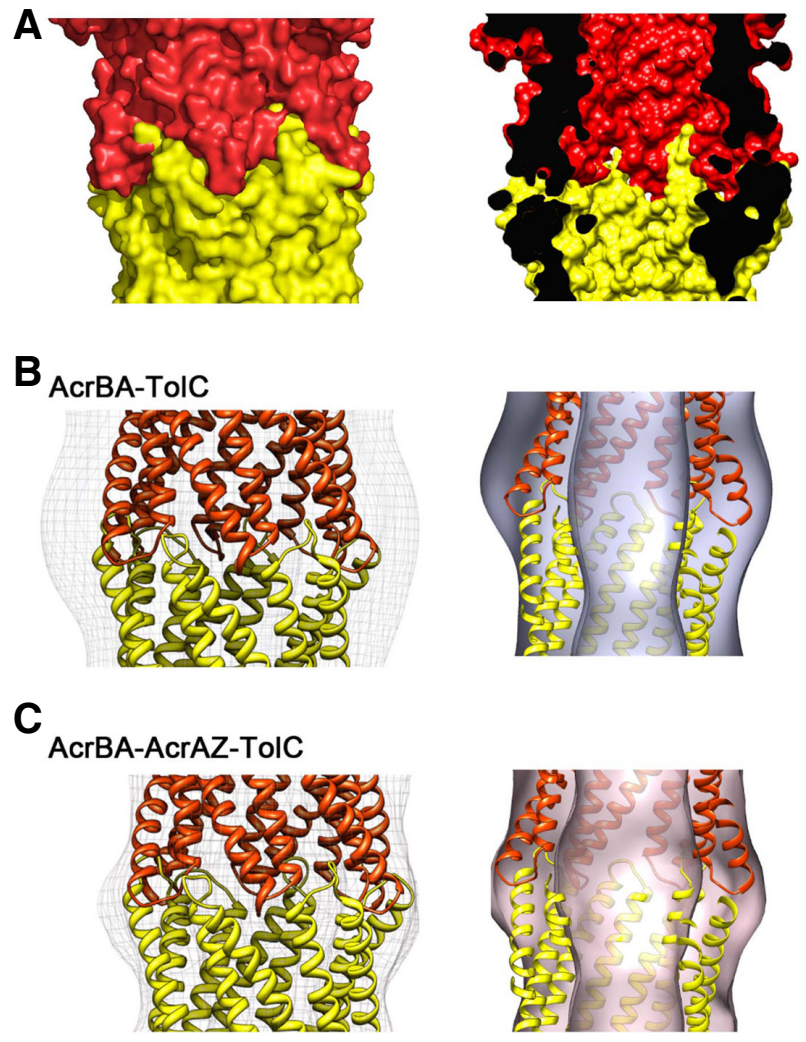

Fig. 4. Intermeshing cogwheel interactions between the AcrA hexamer and TolC trimer in the open state. (A) The surface representative model (left) and its section along the symmetry axis (right) of the binding interface between the AcrA hexamer and TolC trimer based on the intermeshing cogwheel interactions. (B) The complex model and the section of the AcrA hexamer and TolC trimer, which is docked into $3 D$ reconstructions of the AcrBA fusion protein and TolC (AcrBA-TolC) described in Fig. 2. (C) The complex model is docked into the cryo-EM density map reported in Du et al. (2014) (AcrBA-AcrAZ-TolC; EMDB ID: EMD-5915) The EM maps are shown in surface, the AcrA hexamer is colored yellow, and TolC is in red.

Electron microscopy analysis of the AcrBA fusion protein and TolC $\alpha$-hairpin tip region

Although the AcrBA fusion protein and TolC EM density map provide an important implication for the AcrAB-TolC pump assembly, how TolC in the fully-open structure interacts with AcrA remains unclear due to the limited resolution of the EM map. Prior to this studv, we investiqated the fullv-opened structure of TolC usina a chimeric protein from $A$. actinomvcetemco- mmitans $(\mathrm{Aa}), \mathrm{MacA}$, that contains TolC $\alpha$-barrel tip region, which we referred to as MacA-TolC $\alpha$-hybrid-dimer (Xu et al., 2011a; 2011b; 2012). Because the MacA-TolC $\alpha$-hvbrid-dimer protein exhibited a strong affinity to MacA and AcrA in vitro [Kd value of $\sim 0.7 \mathrm{nM}$ (Xu et al., 2011b)], this hybrid protein was considered to exhibit a TolC structure that specifically binds AcrA or MacA. To test whether the hybrid protein represented the fully opened TolC structure for binding to the AcrBA complex, we produced a protein complex comprising the AcrBA fusion protein as well as this hybrid protein and performed a similar electron microscopic analysis.
The asymmetric dumb-bell shaped particles were identified in negative-staining EM images, which were processed to create the 3D-reconstitution at a $24 \AA$ resolution (Figs. $3 A$ and $3 B$ ). The overall structure and size were similar to the AcrBA fusion protein and TolC. Compared with the dumb-bell structure of the AcrA (or MacA) and the MacA-TolC $\alpha$-hybrid-dimer protein complexes previously reported (Xu et al., 2011a; 2011b; 2012), this complex structure is similar, except for the AcrB, which generated asymmetry in the dumb-bell structure. The AcrB trimer was docked to the binary complex model comprising the AcrA hexameric model and MacA-TolC $\alpha$-hybrid-dimer using the complex comprising the AcrBA fusion protein and TolC as a reference (Fig. $3 \mathrm{C}$ ). Because this AcrBA and MacA-TolC hybrid protein complex structure is consistent with the AcrBA fusion protein and TolC complex, we conclude that the binding interface between AcrA and the TolC $\alpha$-barrel tip region in this study as well as in previous reports using the MacA-TolC $\alpha$-hybriddimer protein represent the actual AcrAB-TolC pump assembly (Xu et al., 2011a; 2011b; 2012). In particular, the TolC trimer $\alpha$ barrel tip region is twisted by approximately $45^{\circ}$ similar to the corresponding region of the AcrA (or MacA) hexamer to for the intermeshing cogwheel interaction with the AcrA cogwheel in this binding model and the previous EM studies (Fig. 3D) (Xu et al., 2011a; 2011b; 2012). Combined with the AcrBA - TolC structure, this structure largely confirms the adaptor bridging model based the intermeshing cogwheel interaction between AcrA and TolC as well as the fully-opened TolC structure.

\section{DISCUSSION}

In this study, we constructed an AcrBA fusion protein because the components bind with low affinity, and we present the EM structures for the AcrAB-TolC pump assembly using the fusion proteins. The AcrAB-TolC pump structure exemplifies 'adaptor bridging model', which is an alternative to the prevailing 'adaptor wrapping model'.

Du et al. (2014) recently published a structure of the AcrABTolC pump using a different combination of chimeric and fusion proteins. They co-expressed the AcrB-AcrA chimeric protein and AcrA-AcrZ fusion protein, which showed partial activity similar to our fusion protein (Du et al., 2014). They produced a crvo-EM densitv map and modeled the components in the electron densitv based on hexameric orqanization of MacA from $A$. acinomvcetemcomitans (Xu et al., 2012; Yum et al., 2009) as well as the interaction between $E$. coli CusA and CusB in the metal-efflux pump (Su et al., 2011). The overall EM map from Du et al. is essentially the same as our map, but higher in resolution. In particular, the EM map exhibited a dilated feature at the binding interface between AcrA and TolC, which was also apparent in our two EM maps. Because this dilated feature at the binding interface is common among the previously reported AcrA (or MacA) and TolC tip region (Xu et al., 2010; 2012; Yum et al., 2009), this feature is not likely due to experimental errors from the relatively low resolution of the EM density maps. The proposed binding mode between AcrA and TolC in Du et al.'s docked model differs from our model. In their model, the AcrA $\alpha$-barrel structure cogwheel shows a flared conformation similar to the $\alpha$-hairpin end region of CusB structure complexed with CusA (Su et al., 2011), which leads to widening of the AcrA $\alpha$ barrel. The TolC $\alpha$-barrel was partly inserted into the wider AcrA $\alpha$-barrel. Due to these structural feature, we call this model 'adaptor partial wrapping model'. According to 'adaptor partial wrapping model', the TolC channel should be narrowed at the binding interface between AcrA and TolC. However, neither 
their EM density map nor ours shows the narrowed channel at the binding interface. Both density maps rather display the dilated channel at the binding interface (Fig. 4). Thus our docking model based on the intermeshing cogwheel interaction between AcrA and TolC appears to be more consistent with the EM density map (Fig. 4).

Our docking model based on the intermeshing cogwheel interaction better accounts for the previously reported biochemical and genetic results. The three conserved residues at the $\alpha-$ hairpin tip region of the adaptor proteins were crucial for binding to the TolC $\alpha$-hairpin tip region (Xu et al., 2010; Yum et al., 2009); vice versa, 24 amino acids in the TolC $\alpha$-hairpin tip region were sufficient to bind the adaptor proteins (Lee et al., 2012; Xu et al., 2011a; 2012). However, further high resolution structures are necessary to discern the binding assembly between AcrA and TolC as well as determine how the TolC channel is opened. In this study, we present structural features of the AcrAB-TolC pump, which shows the adaptor bridging model based on the intermeshing cogwheels interaction between the AcrA hexamer and TolC trimer. Because binding between the components is crucial to the multidrug efflux pumps, compounds that inhibit protein-protein interactions between the components are good candidates for preventing multidrug resistance in pathogenic bacteria.

Note: Supplementary information is available on the Molecules and Cells website (www.molcells.org).

\section{ACKNOWLEDGEMENTS}

This study was supported by grants from the Korean Healthcare Technology R\&D Project, Korean Ministry of Health and Welfare (Grant no. H12C0947) and Korea Basic Science Institute (Grant T34518).

\section{REFERENCES}

Du, D., Wang, Z., James, N.R., Voss, J.E., Klimont, E., OheneAgyei, T., Venter, H., Chiu, W., and Luisi, B.F. (2014). Structure of the AcrAB-TolC multidrug efflux pump. Nature 509, 512-515.

Emsley, P., and Cowtan, K. (2004). Coot: model-building tools for molecular graphics. Acta Crystallogr. D Biol. Crystallogr. 60, 2126-2132.

Fralick, J.A. (1996). Evidence that TolC is required for functioning of the Mar/AcrAB efflux pump of Escherichia coli. J. Bacteriol. 178, 5803-5805

Goddard, T.D., Huang, C.C., and Ferrin, T.E. (2007). Visualizing density maps with UCSF Chimera. J. Struct. Biol. 157, 281-287.

Hinchliffe, P., Symmons, M.F., Hughes, C., and Koronakis, V. (2013). Structure and operation of bacterial tripartite pumps. Annu. Rev. Microbiol. 67, 221-242.

Janganan, T.K., Bavro, V.N., Zhang, L., Matak-Vinkovic, D., Barrera N.P., Venien-Bryan, C., Robinson, C.V., Borges-Walmsley, M.I., and Walmsley, A.R. (2011). Evidence for the assembly of a bacterial tripartite multidrug pump with a stoichiometry of 3:6:3. J. Biol. Chem. 286, 26900-26912.

Kim, H.M., Xu, Y., Lee, M., Piao, S., Sim, S.H., Ha, N.C., and Lee, K. (2010). Functional interrelationships between the AcrA hairpin tip region and the TolC aperture tip region for the formation of bacterial tripartite efflux pump AcrAB-TolC. J. Bacteriol. 192, 4498-4503.

Koronakis, V., Sharff, A., Koronakis, E., Luisi, B., and Hughes, C. (2000). Crystal structure of the bacterial membrane protein TolC central to multidrug efflux and protein export. Nature 405, 914919.

Lee, M., Jun, S.Y., Yoon, B.Y., Song, S., Lee, K., and Ha, N.C. (2012). Membrane fusion proteins of type I secretion system and tripartite efflux pumps share a binding motif for TolC in gram-negative bacteria. PLoS One 7, e40460.

Lewis, K. (2000). Translocases: a bacterial tunnel for drugs and proteins. Curr. Biol. 10, R678-681.

Lobedanz, S., Bokma, E., Symmons, M.F., Koronakis, E., Hughes, C., and Koronakis, V. (2007). A periplasmic coiled-coil interface underlying TolC recruitment and the assembly of bacterial drug efflux pumps. Proc. Natl. Acad. Sci. USA 104, 4612-4617.

Ma, D., Cook, D.N., Alberti, M., Pon, N.G., Nikaido, H., and Hearst, J.E. (1993). Molecular cloning and characterization of acrA and acrE genes of Escherichia coli. J. Bacteriol. 175, 6299-6313.

Martins, A., and Amaral, L. (2012). Screening for efflux pump systems of bacteria by the new acridine orange agar method. In Vivo 26, 203-206.

Mikolosko, J., Bobyk, K., Zgurskaya, H.I., and Ghosh, P. (2006). Conformational flexibility in the multidrug efflux system protein AcrA. Structure 14, 577-587.

Mima, T., Joshi, S., Gomez-Escalada, M., and Schweizer, H.P. (2007). Identification and characterization of TriABC-OpmH, a triclosan efflux pump of Pseudomonas aeruginosa requiring two membrane fusion proteins. J. Bacteriol. 189, 7600-7609.

Morshed, S.R., Lei, Y., Yoneyama, H., and Nakae, T. (1995). Expression of genes associated with antibiotic extrusion in Pseudomonas aeruginosa. Biochem. Biophys. Res. Commun. 210, 356-362.

Murakami, S., Nakashima, R., Yamashita, E., and Yamaguchi, A. (2002). Crystal structure of bacterial multidrug efflux transporter AcrB. Nature 419, 587-593.

Narita, S., Eda, S., Yoshihara, E., and Nakae, T. (2003). Linkage of the efflux-pump expression level with substrate extrusion rate in the MexAB-OprM efflux pump of Pseudomonas aeruginosa. Biochem. Biophys. Res. Commun. 308, 922-926.

Nikaido, H., and Zgurskaya, H.I. (1999). Antibiotic efflux mechanisms. Curr. Opin. Infect Dis. 12, 529-536.

Rouquette-Loughlin, C.E., Balthazar, J.T., and Shafer, W.M. (2005). Characterization of the MacA-MacB efflux system in Neisseria gonorrhoeae. J. Antimicrob. Chemother 56, 856-860.

Seeger, M.A., Schiefner, A., Eicher, T., Verrey, F., Diederichs, K., and Pos, K.M. (2006). Structural asymmetry of AcrB trimer suggests a peristaltic pump mechanism. Science $313,1295-$ 1298.

Stegmeier, J.F., Polleichtner, G., Brandes, N., Hotz, C., and Andersen, C. (2006). Importance of the adaptor (membrane fusion) protein hairpin domain for the functionality of multidrug efflux pumps. Biochemistry 45, 10303-10312.

Su, C.C., Long, F., Zimmermann, M.T., Rajashankar, K.R., Jernigan, R.L., and Yu, E.W. (2011). Crystal structure of the CusBA heavy-metal efflux complex of Escherichia coli. Nature 470, 558-562.

Symmons, M.F., Bokma, E., Koronakis, E., Hughes, C., and Koronakis, V. (2009). The assembled structure of a complete tripartite bacterial multidrug efflux pump. Proc. Natl. Acad. Sci. USA 106, 7173-7178.

Tamura, N., Murakami, S., Oyama, Y., Ishiguro, M., and Yamaguchi, A. (2005). Direct interaction of multidrug efflux transporter AcrB and outer membrane channel TolC detected via site-directed disulfide cross-linking. Biochemistry 44, 1111511121.

Tang, G., Peng, L., Baldwin, P.R., Mann, D.S., Jiang, W., Rees, I., and Ludtke, S.J. (2007). EMAN2: An extensible image processing suite for electron microscopy. J. Struct. Biol. 157, 3846.

Touze, T., Eswaran, J., Bokma, E., Koronakis, E., Hughes, C., and Koronakis, V. (2004). Interactions underlying assembly of the Escherichia coli AcrAB-TolC multidrug efflux system. Mol. Microbiol. 53, 697-706.

Trepout, S., Taveau, J.C., Benabdelhak, H., Granier, T., Ducruix, A. Frangakis, A.S., and Lambert, O. (2010). Structure of reconstituted bacterial membrane efflux pump by cryo electron tomography. Biochim. Biophys. Acta 1798, 1953-1960.

Xu, Y., Sim, S.H., Nam, K.H., Jin, X.L., Kim, H.M., Hwang, K.Y., Lee, K., and Ha, N.C. (2009). Crystal structure of the periplasmic region of MacB, a noncanonic ABC transporter. Biochemistry 48 , 5218-5225

Xu, Y., Sim, S.H., Song, S., Piao, S., Kim, H.M., Jin, X.L., Lee, K., and Ha, N.C. (2010). The tip region of the MacA alpha-hairpin is important for the binding to TolC to the Escherichia coli MacABTolC pump. Biochem. Biophys. Res. Commun. 394, 962-965.

Xu, Y., Lee, M., Moeller, A., Song, S., Yoon, B.Y., Kim, H.M., Jun, 
S.Y., Lee, K., and Ha, N.C. (2011a). Funnel-like hexameric assembly of the periplasmic adapter protein in the tripartite multidrug efflux pump in gram-negative bacteria. J. Biol. Chem. 286, 17910-17920.

Xu, Y., Song, S., Moeller, A., Kim, N., Piao, S., Sim, S.H., Kang, M., Yu, W., Cho, H.S., Chang, I., et al. (2011b). Functional implications of an intermeshing cogwheel-like interaction between TolC and MacA in the action of macrolide-specific efflux pump MacAB-TolC. J. Biol. Chem. 286, 13541-13549.
Xu, Y., Moeller, A., Jun, S.Y., Lee, M., Yoon, B.Y., Kim, J.S., Lee, K., and $\mathrm{Ha}$, N.C. (2012). Assembly and channel opening of the outer membrane protein in tripartite drug efflux pumps of Gramnegative bacteria. J. Biol. Chem. 287, 11740-11750. Yum, S., Xu, Y., Piao, S., Sim, S.H., Kim, H.M., Jo, W.S., Kim, K.J., Kweon, H.S., Jeong, M.H., Jeon, H., et al. (2009). Crystal structure of the periplasmic component of a tripartite macrolidespecific efflux pump. J. Mol. Biol. 387, 1286-1297. 